\section{UTERINE HEMORRHAGE OF BENIGN ORIGIN TREATED BY IRRADIATION}

\section{AN ANALYSIS OF FIVE HUNDRED AND TWENTY- SEVEN CASES OF MYOMA UTERI AND MYOPATHIC LESIONS *}

\author{
JOHN G. CLARK, M.D. \\ AND \\ FLOYD E. KEENE, M.D. \\ PHILADELPHIA
}

We now have available for statistical study 527 cases of myomas and other benign lesions causing uterine hemorrhages which have been treated by irradiation in the gynecologic department of the university hospital. In various publications, we have defined from time to time the indications for and contraindications to this treatment; and we have carefully followed a set of rules which clinical experience has taught us are essential safeguards. The patient who falls into the hands of a radiologist who is not a surgeon, or the surgeon who does not employ this valuable remedy as part of his armamentarium, is indeed riding on perilous waters; for neither irradiation nor surgery can be employed exclusively if the best interests of the patient are to be served. Radium in the treatment of uterine hemorrhage of benign origin is chiefly applicable to patients in the fourth and fifth decades of life; but with proper precautions and in carefully selected cases, it may be employed successfully in younger women.

To bring this issue out more clearly, we shall discuss this treatment from the standpoint of age, and we have classified these patients as follows: adolescent girls and young women up to the age of 20 ; young women from 20 to 35 ; middle aged women from 35 to 45 , and those entering the climacteric years.

\section{ADOLESCENT GIRLS AND YOUNG WOMEN}

Occasionally, without definable cause, young girls either menstruate excessively, the flow at times amounting to a more or less persistent metrorrhagia, which in many instances may not interfere greatly with health, but, on the other hand, may finally reduce the patient to a severe degree of anemia. Quite naturally, this condition may be a simple functional derangement incident to the inauguration of the menses, or it may be due to some more or less obscure cause, which is difficult to ascribe to a demonstrable patholgic lesion. Such cases usually tend to self-correction under appropriate hygienic and medical treatment; therefore, we studiously avoid any direct gynecologic intervention until the patient's health is manifestly deteriorating. Since the cooperation between the ovarian impulse and menstrual function is so interdependent, our inclination has been to ascribe these irregularities to some deviation of ovarian function, since a curettage so seldom proves more than temporarily effective. But one should be most wary in using radium in adolescent patients on the very threshhold of young womanhood, because in sufficient dosage it will just as surely stop menstruation for all time as will double ovariotomy.

In no field, therefore, should this agent be used with such precautions, and to this end we hold the initial irradiation at a very small dosage, with the possibility of only partial relief or even failure, with a view to a

\footnotetext{
* Read before the Section on Obstetrics, Gynecology and Abdominal
} Surgery at the Seventy-Third Annual Session of the American Medical Association, St. Louis, May, 1922. second irradiation, rather than resort at once to a more prolonged exposure with its dangers. In these young persons, after an examination under anesthesia for the purpose of accurately defining the pelvic conditions, we may with safety apply $50 \mathrm{mg}$. for from three to four hours. This usually proves effective in regulating the periods; but we never reach any conclusion as to its failure to cure under six months, for we fear the effects of a too early repetition of the treatment. Should the second irradiation be necessary, the first having been partially or wholly ineffective, we then increase the time from four to six hours, depending to some extent on the size of the uterus and the severity of the bleeding. In our experience, the second application is always effective.

Fortunately, these cases are of infrequent occurrence; hence, the very small number in our list. Precisely the same contraindication must influence one here as in all other cases; namely, in the presence of any inflammatory involvement of the tubes, irradiation is very postively contraindicated. Occasionally, a small cyst, or tuberculosis of the endometrium may be the hemorrhagic genesis. It is especially important, therefore, to exclude every possible cause for the metrorrhagia that might contraindicate the use of radium. In our series of 527 cases, there have been only five treated between the ages of 11 and 20 years. All of these patients except one were cured by the first treatment. One girl of 11 had $50 \mathrm{mg}$. applied for three hours; two, aged 15 had eight and five hour applications, respectively; two, aged. 17, five and eight hours, respectively. One of these who was subjected to an eight-hour application suffered with flushes for one year-too perilously close to a menopause for comfort.

\section{YOUNG WOMEN}

In the cases of women, aged from 20 to 35 , myomatous changes in the uterus begin to show their incidence in an increasing ratio, very few appearing between 20 and 25, but from then on gradually becoming more frequent. In women of this age, two controlling motives in life should be kept in the foreview: (1) her maternal possibilities, and (2) the sexual life. While radium is most effective in inhibiting the growth and ultimately diminishing the size of the tumor during these ages, it is also equally powerful in inducing a premature menopause. Therefore, the patient should be studied from the standpoint of a myomectomy if possible, as this operation is unquestionably to be preferred; and should this not be feasible, we are, as a rule, favorably inclined to a subtotal hysterectomy, with the preservation of the ovaries. In the first instance, the sexual and maternal physiology are not interfered with, but rather promoted; while in the second, menstruation may continue and the sexual life is in no way disturbed.

With this disposal of myomas occurring in young women, it will be noted that surgical intervention takes decided precedence over that of irradiation, leaving relatively few cases open to the latter treatment. As a result of our experience, we usually restrict the dosage of radium to $50 \mathrm{mg}$. for from six to twelve hours in these younger women. In such instances, the menstrual periods may recur more or less normally after a variable period of amenorrhea. Seventy-seven patients falling within these ages were subjected to irradiation. The age distribution was as follows: from 20 to 25 , eleven; from 25 to 30 , eighteen; from 30 to 35 , forty-eight. 


\section{MIDDLE AGED WOMEN}

In the group of women aged from 35 to 45 the question of the maternal possibilities as well as the sexual life of the person becomes of much less importance in the choice of treatment. Usually, the maternal equation is either settled by the patient having a family, or the myomatous changes in the uterus have so distorted its structure as to render the patient sterile, or have made childbearing impossible. Further, the cessation of the ovarian function in women who have established sexual habits is of far less moment in its influence on this function, for the libido sexualis is now largely dependent on the psychic side of the individual rather than on the centers in the lower spinal cord, which are of capital influence in the inauguration of the sexual instincts.

If either of these phases stand out prominently in the patient's mind and she fears the abrogation of either, we incline toward surgical intervention. The ultimate happiness of the patient must have equal consideration with the restoration of physical health; for the latter without the former may have little merit in the mind of a patient suffering under a cloud of such misgiving. In such cases, a follow-up letter does not bring a very cheerful or grateful response, even though the patient's life may actually have been saved.

However, in considering the use of radium, we find that this question has largely been settled by the fact that the patient has passed the fortieth year. At this period, therefore, the preference, in case of doubt, always falls in favor of radium; whereas, in the younger women, surgery is almost invariably the remedy chosen.

\section{WOMEN IN THE CLIMACTERIC YEARS}

In the group of cases formed by women in the climacteric years, radium occupies a premier position; it is effective, it is quick in giving relief, the treatment does not incapacitate the patient, it is safe, and it is followed by practically no immediate disabling sequelae or remote ill effects, as our summary will show. With our experience as a guide, we have ceased to summarize what may happen from three to five years after irradiation; for, without exception thus far, we have nothing to record in the way of so-called continuing or remote changes which may be counted as a backflare from the treatment.

This statement is now well grounded on the careful study of cases running back six years. The fear of sarcomatous transformation of the tumor is without foundation, for in not one single instance has this malignant change been noted after irradiation in this large series. Hence, any criticism of radium treatment on this false assumption is without support. The cure in these cases is prompt, and the only after-effects are those noted in all women passing through the menopause: Many pass through this trying period with scarcely a complaint; others are troubled constantly; while a few have a very stormy and occasionally, a prolonged period of adjustment. At times, we have thought that possibly a menopause induced by irradiation is more violent than in other series of cases in which the climacterium is surgically induced; but when a large series of patients who have undergone double ovariotomy is compared with those who have been stubmitted to irradiation, the two are found to be similar.

In a review by Norris and Clark of two comparative series of myomatous and of pelvic inflammatory cases, in which the ovaries were preserved or removed, Norris, after a very close scrutiny of the results according to the age incidence, brought out a rather startling and revolutionary point which is fully substantiated in this study. In a large group of young women who have undergone double ovariotomy, there will unquestionably be a greater percentage who suffer acutely from climacteric disturbances; but it is strikingly evident that, in an equal number of patients either within or actually beyond the normal menopausal years, there will also be a considerable percentage in which these disturbances are quite as stormy and equally prolonged.

The temperamental and the nervous stability of these women has an infinitely greater bearing on this critical epoch than the mere question of age. For this reason, we study much more attentively than formerly the nervous status of the patient, when this decision as to the artificial induction of the menopause is under consideration. This is particularly to be taken into full account in young women; whereas, those who are within the menopausal years may be treated as the pathologic status indicates. This observation of Norris we especially emphasize, for it is of capital importance and must be carefully heeded if the best postoperative results are to be achieved.

\section{SUMMARY OF RESULTS IN FIVE HUNDRED AND TWENTY-SEVEN CASES}

The total number of cases of benign hemorrhage of the uterus occurring in myoma uteri and myopathic lesions is 527 . Of this number, there are available for statistical study 476 cases which have been fully treated; of the remaining fifty-one, some have not been heard from and others have been too recently treated for conclusive study.

After irradiation, it is common for one or two and rarely three menstrual periods to occur before the actual onset of the permanent amenorrhea. To some extent, the time of the application of radium relative to the menstrual epoch influences the succeeding periods. If radium is applied shortly before the expected period, it is not likely in any way to influence the succeeding period; to the contrary, it may actually be more profuse; but, we may assume that within from four to six weeks at most the structural changes induced by the irradiation will have progressed sufficiently to stop the menstrual flow entirely.

In 476 cases which required only one treatment, permanent amenorrhea occurred promptly, or, after a short interval of temporary amenorrhea, there was a return to normal flow, when the dosage had been intentionally light with this end in view. Of 433 cases, forty either required a second application of radium, or an operation was necessitated on account of a persistent flow. The actual percentage in which the bleeding was controlled by the first application of radium in 476 cases was 91 per cent. In this entire number, only one death occurred, and this was of obscure origin. The patient was very stout, and within twenty-four hours after a plastic operation on the vagina, accompanied with a simple dilation and curettage, and an intra-uterine application of $50 \mathrm{mg}$. of radium, she developed severe pains in the epigastric region, which soon defined itself as a general peritonitis, from which she died.

A necropsy was not permitted in this case, and we are at a loss to know how to account for the fatality, particularly as the initial symptoms began in the upper abdomen and not in the region of operation. We are 
inclined to think that there was a second lesion in the upper abdomen, and the ultimate catastrophe may have resulted from the perforation of an ulcer or possibly from some accident incident to a gallbladder or pancreatic lesion.

\section{RECURRENCE OF BLEEDING AFTER IRRADIATION}

In forty-seven cases, bleeding recurred after the application of radium; in twenty-seven, several months after the treatment. The remainder were noted as follows: one year subsequently, nine cases; from one to one and one-half years, two cases; from one and onehalf to two years, three cases; from two to three years, six cases. Of these forty-seven, the recurrence was slight, or the periods returned to normal and did not require subsequent treatment, in twenty-three cases.

\section{LEUKORRHEA}

Frequently, after an intra-uterine application of radium, a thick yellowish leukorrhea develops, which is slight in amount and is seldom if ever persistent, rarely lasting more than from six to ten weeks. Frequently, it is so insignificant that unless the patient's attention is called to it, she does not refer to it. Further, along this line, there is a gratifying sequel in that a persistent leukorrhea is not infrequently cured by the treatment. We, therefore, lay little or no stress on the presence of leukorrhea as a sequel, because it is evanescent in character, and requires little or no attention.

\section{MENOPAUSE}

Irradiation in a dosage of $50 \mathrm{mg}$., for twelve to twenty-four hours, will produce climacteric symptoms of varying degrees of intensity in 60 per cent. of cases. In 36 per cent., the symptoms will be marked or severe. As a rule, they persist for from a few months to two or three years, and then subside. In an occasional case, severe symptoms may persist for several years. Menopausal symptoms seldom if ever occur in young women when a dosage of $50 \mathrm{mg}$. is applied for from six to eight hours; and in no case has the climacterium been induced by this dosage. Larger doses will produce these symptoms; but our impression is that they are usually neither so persistent nor so severe in younger women as those of menopausal years. Strangely, this observation is at variance with the usual conception of the menopause, but it is in line with the statement of Clark and Norris in their analysis of cases after a complete hysterectomy.

From this study, we also draw the conclusion that the age factor in its relationship to the menopause is not nearly so much to be taken into account as the temperamental and nervous factors. Thus, a highly strung woman with poor nervous governors is very likely to be thrown markedly out of balance by the precipitation of the menopause, be it early or late. This point we would especially emphasize.

\section{PREGNANCY}

As the vast majority of these patients were subjected to irradiation after childbearing possibilities had ceased either through age limitation or because of the distortion of the uterus incident to pathologic changes, the consideration of pregnancy has but little bearing as a deciding factor in the choice of treatment. As we have already indicated in the preceding pages, our inclination is to perform an operation in the younger women rather than to use radium.

In our total number of cases in which radium has been used, there have been five women who subse- quently became pregnant. In one, a woman, aged 29 , suffering with persistent menorrhagia of a myopathic character, in whom a three hour application of $85 \mathrm{mg}$. of radium was employed, the menses returned to normal after three preceding curettages had failed to effect this regulation. Two years after marriage, a full term healthy child was born. The second patient, aged 31, had suffered from excessive menorrhagia for ten years, induced by a myomatous tumor the size of a small orange. Subsequent to the irradiation with $50 \mathrm{mg}$. for twenty-four hours, she passed through a definite menopausal cycle. The periods then came back to normal for a short time and pregnancy occurred. She passed through a normal pregnancy up to full term, but succumbed to a postpartum hemorrhage, following the birth of the baby by accouchement forcé. In a third patient, pregnancy occurred two years after an application of $50 \mathrm{mg}$. for twenty-four hours, but resulted in a miscarriage. In a fourth, a normal delivery occurred after three years in a patient who had been submitted to an application of $50 \mathrm{mg}$. of radium for six hours. In a fifth patient, who had twice received applications of $25 \mathrm{mg}$. of radium for twenty-four hours, three pregnancies occurred. All of these children were born prematurely and were either dead at delivery or died subsequently.

From this summary of results, it is very plainly evident that the application of radium gives but little forecast in favor of restoring the childbearing possibilities; for, of these seven pregnancies, only two children came to full maturity. This study, therefore, emphasizes the statement already laid down that when pregnancy is still in contemplation by the patient, an operation should be advised, instead of irradiation.

\section{LATE RESULTS OF IRRADIATION}

The criticism offered to the use of radium has been that the final results of irradiation are not yet known. We believe that this skeptical forecast may be set at rest when we find in our large list of cases that, in at least 300 of them that have been followed from three to six years, thus far no single instance has come to our attention in which there was any remote deleterious result which could in any way be traced to radium.

In one instance, a patient who was operated on in another clinic after irradiation by us was found to have a carcinoma of the fundus. Previous to the treatment by us, a curettage had been done by a competent gynecologist and a negative report as to malignancy had been returned from his laboratory. The bleeding recurred after the curettage, and the patient then came under our care. The second curettage brought away insufficient débris for a pathologic examination. The hemorrhages had been absolutely periodic in character, the uterus was slightly enlarged, and there were no grounds on which to base a diagnosis of malignancy, all the evidence being in favor of a benign source of hemorrhage. Irradiation in this case caused a cessation of hemorrhage for sevaral months, when it again recurred; and, at an operation in another hospital, an extensive carcinoma of the fundus was found. Whether there was an oversight at the time of the irradiation, or whether the carcinoma originated subsequently, is open to question. Admitting the probabilities that the carcinoma was overlooked at the time of irradiation, this was but one single instance in 527 cases of such an oversight; and, as we review our 96 per cent. of permanent cures, we do not feel that this is a very serious indictment against irradiation, partic- 
ularly as this error did not preclude the subsequent hysterectomy.

FAILURE TO RELIEVE BY ONE IRRADIATION

A reapplication was necessary for the relief of a continuation or a recurrence of the abnormal bleeding in twenty-eight cases. In all of these, the condition was cured by a second application, except in two, which required operation. The types of cases requiring a second treatment were: myomas, eight cases; myopathic hemorrhage, five cases; endometritis, two cases; bleeding from cervical stump, one case; pelvic inflammatory disease, one case; not stated, one case.

Of this list, an error was committed in reapplying radium in the pelvic inflammatory case, notwithstanding the fact that the bleeding was controlled and the patient was relieved of the symptoms; for inflammatory cases should not be submitted to irradiation. Further, it may be said of the twenty-eight patients that thirteen were subjected to $50 \mathrm{mg}$. of radium from six to twelve hours. In other words, in this list there were several young women in whom the light application of radium was made with the probability of having to resort to a second treatment.

\section{CASES REQUIRING OPERATION AFTER IRRADIATION}

Of the 527 patients, a total of eighteen were operated on subsequent to irradiation. Fourteen of these were myomatous cases in which the bleeding persisted or recurred more or less profusely following a variable period of amenorrhea. Four patients were subsequently operated on for chronic pelvic inflammatory clisease and one for hemorrhage. It is gratifying to note that in this long list of cases, only 3 per cent. of the patients required operation after irradiation: certainly a very small percentage in a series of cases of so many varied conditions.

In the fourteen patients requiring a subsequent operation, ten of the operations were for myomas of the larger submucous type, three for myopathic hemorrhage, and one, in which there was recurrent bleeding from a cervical stump after a hysterectomy, for an aclenomyoma.

\section{MORTALITY}

In the entire series, there was but one death, which occurred from peritonitis a few days after an extensive plastic operation and an application of $50 \mathrm{mg}$. of radium for twenty-four hours. The postoperative symptoms were those of an acute upper abdominal lesion. Necropsy was not performed.

\section{COMPLICATIONS OF IRRADIATION}

Phlebitis occurred in two cases; in one, a mere recurrent symptom of an old phlebitis. In the second, bilateral phlebitis followed on a repair of the cervix, a posterior colporrhaphy and irradiation. Eight patients developed symptoms of more or less severe neuritis, which is not an infrequent occurrence in women at the menopause, regardless of any antedating treatment or operation.

\section{TECHNIC}

The technic of application is very simple. Under nitrous oxid and oxygen anesthesia, a careful pelvic examination is made. The size of the uterus is ascertained, and its depth is measured by a sound and recorded for future reference. A thorough curettage is performed, and the curettings are saved for microscopic diagnosis. A $50 \mathrm{mg}$. tube of radium properly filtered is inserted to the fundus of the uterus. The duration of application depends largely on the age of the patient; in women at or near the menopause, the duration is usually twenty-four hours, and in young women proportionally less. A repetition of the application in young women is preferable to overirradiation.

A point which we always emphasize in the use of radium in all cases of benign hemorrhage is that one intra-uterine application of $50 \mathrm{mg}$. for twenty-four hours suffices to cure the case effectively. We, therefore, strongly deprecate the repetition of these treatments, since each application carries a minimum danger with it, and has no merit whatever over one application which effectively cures. Also, we do not favor coincident roentgen-ray treatments, for they are quite unnecessary.

The immediate results of irradiation are not unlike those of a simple curettage, with the exception that nausea is likely to be more persistent during the retention of the radium within the uterus. In all of this list, we have had no case of vomiting so persistent or prolonged as to give the slightest cause for anxiety. Usually, within one or two hours at most after the withdrawal of the radium, the nausea and vomiting cease. In a few instances, these symptoms have persisted for three days. As a rule, these patients remain in the hospital five days; for we follow the rule here that we observe in any case of simple curettage. It is unwise to permit the patient to go home the second day after irradiation, since the surgeon must see the patient through any possible danger arising from his surgical intervention, and such dangers are not past within twenty-four hours. As a rule, the patient sits up the third day and leaves the hospital the fifth day.

The standing rule is for all of these patients to return for examination at the end of from six weeks to two months after the treatment. If the patient lives at a distance and cannot return, a personal letter as to her condition is requested of the patient or of the attending physician. From our study of these final results, we feel that a three-year period suffices for a complete observation. By no means do all tumors disappear, and a few do not diminish greatly in size; but as the majority of these cases have been treated solely for hemorrhages, with the cessation of this symptom the patient may be looked on as cured. After irradiation, the great majority of tumors either shrink greatly in size or disappear completely. A subperitoneal tumor is not likely to be greatly influenced by irradiation.

\section{CASES IN WHICH OPERATION WAS PERFORMED} AFTER INEFFECTUAL IRRADIATION

CASE 1.-Myoma. One hundred milligrams of radium was applied for twenty-four hours. Operation was performed two months later. There was no relief of hemorrhage. A large tumor was found.

CASE 2.-Myoma, large. Fifty milligrams of radium was applied for twenty-four hours. There was no effect on bleeding.

CASE 3.-Adenomyoma of cervical stump. Fifty milligrams of radium was applied for twelve hours. There was no relief of hemorrhage.

CASE 4.-Myoma. Fifty milligrams of radium was applied for twelve hours. The periods recurred in normal amount a few months after irradiation.

CASE 5.-Myoma, large. Fifty milligrams of radium was applied for twenty-four hours. Two applications were made, with an interval of ten months. Bleeding was not controlled.

CASE 6.-Myoma. Fifty milligrams of radium was applied for eight hours. Bleeding continued. 
CASE 7.-Myoma. Fifty milligrams of radium was applied for twelve hours. Operation was performed two months later, as bleeding was not controlled.

CASE 8.-Myopathic lesions. Fifty milligrams of radium was applied for twenty-four hours. Operation was performed eight months later for recurrence of bleeding.

CASE 9.-Myopathic lesions. Twenty-five milligrams of radium was applied for six hours. Operation was performed two years later for recurrence of bleeding.

CASE 10.-Myoma. Fifty milligrams of radium was applied for twelve hours. Operation was necessary for recurrence of bleeding.

CASE 11.-Myopathic lesions. Fifty milligrams of radium was applied for four hours. There was persistent bleeding.

CASE 12.-Myoma, large. Fifty milligrams of radium was applied for twenty-four hours. There were two treatments, with an interval of nine months. After the second treatment, there was amenorrhea for two years, then a recurrence of bleeding.

CASE 13.-Myoma. Fifty milligrams of radium was applied for twenty-four hours. The tumor was large. There was recurrence of bleeding after nine months, requiring operation.

CASE 14.-Fifty milligrams of radium was applied for fourteen hours. Radium had no effect on bleeding. Operation was performed six months later.

\section{CASES IN WHICH OPERATION WAS PERFORMED FOR SUBSEQUENT PELVIC INFLAMMATORY DISEASE}

CASE 15.-Operation was performed six weeks after irradiation. This was a case of chronic pelvic inflammatory lesion, lighted up by irradiation.

CASE 16.-The patient was well for three years following irradiation, and then developed symptoms of acute pelvic infection of streptococcus type.

CASE 17.-Operation was performed one and one-half years after radium treatment for extensive pelvic inflammation.

CASE 18.- Radium was applied in this case which had been diagnosed as pelvic inflammatory disease at the time of the first treatment. This was a cardinal error, for this treatment is not suited to pelvic inflammatory lesions.

Having been among the first to adopt this plan of treatment following the pioneer work of Drs. Abbe and Kelly, we have endeavored to thread our way cautiously, expanding the field of application only as our experience pointed the way, and we now feel that we are on a solid therapeutic foundation. Following are clinical rules which have been evolved from our observations of this large series of cases.

\section{CONTRAINDICATIONS}

1. Tumors larger than the size of a four months pregnant uterus, or those complicated by inflammatory lesions or neoplasms of the adnexa, should not be submitted to irradiation. Hysterectomy offers quicker relief for the first, and surgical intervention is imperatively indicated in the second class. In exceptional cases, large tumors have been successfully irradiated in . the face of some grave contraindications to the major operation, and in others this treatment has been employed to check hemorrhage in seriously anemic patients, thus permitting a sufficient recuperation to insure a safer operation.

2. In tumors causing pressure symptoms, the shrinkage of the tumor is too slow after irradiation; therefore, hysterectomy is to be preferred.

3. When, in tumors, there is cachexia out of proportion to the blood loss, this may be due to a necrosis of the tumor, which may be hastened by the irradiation. We have observed such instances. The recovery of the patient is very prompt and most satis- factory after hysterectomy, and it is, therefore, preferable.

4. The larger submucous tumors, and those so distorting the cervical canal and fundal cavity as to render the introduction of the radium capsule difficult or impossible, are not suitable for irradiation. A pyometrium may follow the application of radium in such cases. This sequel has followed in two instances.

5. Tumors rapidly increasing in size or those which have undergone calcareous changes are not adapted to irradiation.

6. Myomas in young women fall, as a rule, within the surgical domain, as a myomectomy or partial hysterectomy, with preservation of the ovarian function, is decidedly preferable.

7. In patients suffering with an associated intraabdominal lesion, such as a cholelithiasis, appendicitis or gastric lesions of surgical consequence, it is injudicious to employ radium. In such instances, an exploratory incision should be made, thus giving a comprehensive diagnostic insight. The abdominal lesion may require first attention; but this does not preclude an irradiation at the same sitting, if the myoma or myopathic cause of the hemorrhage falls within the class to which this treatment is applicable. Under this comprehensive plan, the treatment is fully subservient to the surgeon's judgment.

\section{INDICATIONS FOR IRRADIATION}

From a study of the foregoing contraindications to irradiation, it appears at first glance that the field for its application must be a narrow one; and yet such is not the case. In 1921, 210 patients falling within these two domains were admitted to the gynecologic service of the university hospital. Of these, 110 were submitted to irradiation and 100 to surgical intervention. Both classes of cases, under this careful segregation, yielded first class results. Those undergoing an operation ran greater risks and were longer in the hospital; and the return to efficiency was much slower. But the ultimate outcome was entirely satisfactory, since these patients belonged without question to the surgical field.

We would commit a serious error were we to consider this splendid and most effective plan of treatment as a competitor of surgery. The two go hand in hand, and both must be supervised by the surgeon, and not by the roentgen-ray expert or the roentgenologist, for each in a varying degree is a surgical measure. As to the indications for this treatment, we may briefly summarize it in one sentence: "Irradiation is the treatment of choice for the smaller myomas in women approaching or within the menopausal years whose only symptom is hemorrhage."

$$
2017 \text { Walnut Street-Medical Arts Building. }
$$

\section{ABSTRACT OF DISCUSSION}

Dr. Henry Schmitz, Chicago: In my work I observe the same indications as given by Dr. Clark. In every case of bleeding uterus to be treated with radium I insist on a diagnostic curettage. The ratio between the cases treated with radium and those subjected to surgery is as $1: 3$. In other words, 25 per cent. of so-called benign uterine hemorrhages are treated with radium, and the other 75 per cent. by surgery. Therefore, the treatment of benign uterine hemorrhages belongs to the surgeon and should, under no circumstances, be undertaken by a radiologist unless the latter cooperates with the surgeon. We have treated 142 cases up to date. One patient died from septic peritonitis. The patient was the 
last of five patients being treated on this particular morning with radium for benign and malignant hemorrhages. The same surgeon did the work in the same operating room. The (iagnostic curettage was negative. The bimanual findings were normal. We observed a case of carcinoma of the uterus in spite of careful diagnostic curettage and histologic diagnosis. It was a cervical carcinoma. Hemorrhage recurred six months after the treatment. At this time the cancer had invaded the parametria. Whether the carcinoma was present at the time of the insertion of the radium it is impossible to state, as the microscopic report was negative. It is fair to assume, however, that the patient was suffering with cancer. Another patient returned two years later with an advanced fundus carcinoma, though the diagnostic curettage was microscopically reported as a hypertrophy. Two sarcomatous degenerations were detected very soon after the treatment, and were removed; so the patient did not suffer from procrastination. We also saw four cases of degeneration fibroids : three simple fatty degenerations and one red degeneration. I have mentioned these observations, not to discourage the use of radium in cases of benign hemorrhage, but to bring out the point that it is extremely important to make a very careful diagnostic curettage, and if we are in doubt to resort to surgery rather than procrastinate by the use of radium.

Dr. F. F. Lawrence, Columbus, Ohio: The question seems to be, first. Shall we treat the symptoms? Shall we be satisfied with a symptomatic cure or relief? In some of the cases of radium and roentgen-ray treatment the results are apparently magical, while in others the malignancy seems to be increased in its activity. The first thing we should remember is that every neoplasm, no matter where, is potentially a malignancy. Second, that fibroma or myoma of the uterus, while it develops malignancy, is not carcinoma. It may be the cause of carcinoma by its continued irritation, and produce an epitheliomatous growth which is carcinomatous. With this potential malignancy, we should ask ourselves, Can we afford-when the operation of supravaginal hysterectomy for removal of the myomatous uterus has a mortality, when properly performed, of 1 per cent.-- to deal with this potential malignancy by any type of treatment other than complete removal? Noble and other competent men have shown, and probably Dr. Clark has found, that there is a potential malignancy in fibroma of from 5 to 8 per cent. That is to say, from 5 to 8 per cent. of the fibromas, when removed, have undergone sarcomatous change. When we put against this I per cent. mortality in hysterectomy properly performed, can we afford to run from five to eight times the risk of malignancy by attempting to treat with radium or roentgen ray or anything else?

DR. JoHN G. Clark, Philadelphia: Dr. Schmidt raises the question as to mortality. There was one fatal case among the 527 treated. The death occurred from some obscure cause. The symptoms were of a fulminating character, and occurred in the upper abdomen from apparently some coincident lesion. Neither an operation nor a necropsy was permitted, and we have designated this as a fatality from peritonitis, induced by the curettage, although the symptoms would really not sustain this interpretation. I am, indeed, glad that Dr. Lawrence has brought into the discussion the perennial question of malignant degeneration in myomas. If there was ever a fallacy that should be annihilated, this is one. The frequency of sarcomatous transformations in myomas is so small as almost to be negligible. If, as Dr. Lawrence would have us believe, this peril is so ominous, certainly sarcomatous sequels in the cervical stump after a supravaginal hysterectomy should frequently be noted, and yet in more than 1,500 such operations we have yet to see such a postoperative incident. In our laboratory, in which more than 1,200 uteri have been examined after hysterectomy for myomas, there were only twenty-two sarcomas that were not diagnosed before the specimen was sent to the laboratory. The hiatus in pathologic statistics emanating from different laboratories depends on what one pathologist would call sarcoma and what the other would call fibroma. It is quite possible to take out of almost any fibroid tumor isolated areas which could very easily be mistaken for spindle cell sarcoma. In such instances the whole specimen is of chief diagnostic import, not these compact areas. I am sure, therefore, that the discrepancy in diagnosis lies in this difference in interpretation. We have seen several carcinomas occurring in the cervical stump in which a previous supravaginal hysterectomy has been performed, but a control diagnostic curettage had not been made. As these cases have passed through our clinic, there were 101 cases of adenocarcinoma of the uterus in which the clinical diagnosis was positive in fifty-seven and doubtful malignancy, twenty-four; in the remainder the positive diagnosis was made in the laboratory in cases which otherwise would have been considered of benign type. In such instances proper surgical measures were instituted before the patients were discharged from the hospital. The ultimate judge, therefore, in all of these cases, whether one uses radium or operates, is a skilled pathologist. All too frequently a good general pathologist goes completely amiss on this point, for he may see in normal cyclic glandular changes in the endometrium such puzzling pictures that he may designate them as adenocarcinoma. The main point which I particularly wish to stress is that we should cease to hold over patients the suggestion that a myoma is liable to sarcomatous changes, for such transformations are so small as almost to be negligible and should never be the crucial point on which a radical operation is decided. Dr. Lawrence admits at least a 2 per cent. mortality from surgical intervention. Therefore, in this series, were they all to have been submitted to hysterectomy, there would have been at least ten deaths; under irradiation there has been one.

\section{STANDARDIZATION AND PRESERVATION OF COMPLEMENT SERUM FOR WASSERMANN TEST *}

\section{E. H. RUEDIGER, M.D. BISMARCK, N. D.}

Laboratory workers who are familiar with the Wassermann test know that the serums from different guinea-pigs differ greatly in complement activity, and that under ordinary conditions complement rapidly deteriorates but can be preserved for varying lengths of time under suitable conditions.

About two years ago $I^{1}$ showed that a number of complement serums from guinea-pigs gave results with the same human serum varying from $2+$ to $10+$. Even greater differences have been observed since; serums from some guinea-pigs have given negative results while serums from other guinea-pigs gave $10+$ with the same human serum. Such irregularities can be eliminated by selection of complement serum, probably by selective breeding of guinea-pigs, by selective feeding of guinea-pigs and by preservation of the good complement serrms.

\section{SELECTION OF SERUM AND GUINEA-PIGS}

The preserved positive control serum, composed of a mixture of twenty syphilitic human serums, I diluted to such an extent that it gave approximately $6+$ with a fairly good complement serum. This diluted positive control serum was then used in titrating the complement serums from the different guinea-pigs. At the beginning of this work, about two years ago, the differences in the results were very striking; they varied from negative to $10+$, and from no hemolysis to complete hemolysis. All guinea-pigs whose serums gave less than $5+$ or had poor hemolytic power were rejected and were used for other work or were killed.

\footnotetext{
pital.

* From the pathologic laboratory of the Bismarck Evangelical Hos. 1. Ruediger, E. H.: Journal-Lancet 40:565 (Oct. 15) 1920.
} 